\title{
EQUILÍBRIO DE ADSORÇÃO DE C11 e C12 SOBRE O SAPO-18 E A ZÉÓLITA NaCaA
}

\author{
Diego A. A. Gomes ${ }^{1}$ \\ Silvana Mattedi ${ }^{2}$ \\ Maritza M. Urbina ${ }^{3}$ \\ Antônio O. S. Silva ${ }^{4}$ \\ Luiz A. M. Pontes ${ }^{5}$
}

\begin{abstract}
Resumo: O processo de separação de n-parafinas das ramificadas, naftênicas e aromáticas no refino de petróleo é realizada por meio de adsorção sobre peneiras moleculares. Parafinas lineares possuem um grande interesse de estudo porque são utilizadas na fabricação de diversos produtos, dentre eles o LAB (linear alquilbenzeno), empregado na produção de detergentes biodegradáveis. $\mathrm{O}$ presente trabalho tem como objetivo a obtenção dos dados de equilíbrio de adsorção de parafinas lineares (C11 e C12) sobre o SAPO-18 e a zeólita NaCaA. Os adsorventes foram sintetizados pelo método hidrotérmico e caracterizados através da Difração de Raios-X (DRX), Fluorescência de Raios-X (FRX), Análise Termogravimétrica (TG) e Adsorção de $\mathrm{N}_{2}$ a $-196^{\circ} \mathrm{C}$. A seguir foram obtidas as isotermas de adsorção em banho finito para as parafinas estudadas. As concentrações foram determinadas utilizando um cromatógrafo a gás, equipado com detector FID e coluna capilar tipo OV1. A quantidade adsorvida foi calculada por meio de um balanço de massa.
\end{abstract}

Palavras chave: Adsorção, Parafinas, SAPO-18, Zeólita NaCaA.

Abstract: Linear alkanes of high purity may be recovered from a mixture of linear, branched and aromatic using molecular sieves. Linear paraffins have great interest of study because they are raw material for many products, including the LAB. The Linear alkyl benzenes (LABs) are widely used in the manufacture of emulsifiers and biodegradable detergents. Adsorption equilibrium data of linear alkanes (C11 and $\mathrm{C} 12)$ in the $\mathrm{NaCaA}$ Zeolite and SAPO-18 were determined. The NaCaA Zeolite and SAPO-18 samples were synthesized for hydrothermal method and characterized using $\mathrm{N}_{2}$ adsorption at $-196{ }^{\circ} \mathrm{C}$, TG, XRD and FRX instrumental analytic methods. The analyses were realized in a gas chromatograph (GC) equipped with a flame ionization detector (FID) and OV1 column. The amount adsorbed was obtained by calculating the mass balance for the external liquid and the adsorbed phases.

Keywords: Adsorption, Paraffins, SAPO-18, NaCaA Zeolite.

\footnotetext{
${ }^{1}$ Programa de Pós-Graduação em Engenharia Química, Universidade Federal da Bahia. E-mail: diegoangeloa@yahoo.com.br.

${ }^{2}$ Programa de Pós-Graduação em Engenharia Química, Universidade Federal da Bahia. E-mail: silvana@ufba.br.

${ }^{3}$ Centro de Tecnologia, Universidade Federal de Alagoas. E-mail: maritza@ctec.ufal.br.

${ }^{4}$ Centro de Tecnologia, Universidade Federal de Alagoas. E-mail: osimar@yahoo.com.

${ }^{5}$ Programa de Pós-Graduação em Engenharia Química, Universidade Federal da Bahia, Universidade Salvador e Instituto Brasileiro de Tecnologia e Regulação. E-mail: uolpontes@uol.com.br.
} 


\section{Introdução}

$\mathrm{Na}$ região metropolitana de Salvador, BA, encontra-se a segunda maior refinaria e um dos maiores complexos petroquímicos do Brasil. As características do petróleo originário dessa região o tornam um dos melhores do mundo pelo alto teor de parafinas, baixa acidez e teores de nitrogênio e enxofre. A Refinaria Landulpho Alves (RLAM) utiliza este petróleo para a produção de combustíveis, para produção de nafta petroquímica, lubrificantes e parafinas. As n-parafinas tem maior valor agregado que os combustíveis, e são utilizadas na produção do alquilbenzeno linear (Tejero et al., 2012) e de outras especialidades parafínicas na indústria de terceira geração de petroquímicos, na indústria moveleira e alimentícia (Lira e Tailleur, 2013). O LAB é produzido na DETEN Química, Salvador, BA a partir do n-dodecano obtido na Refinaria com o petróleo característico baiano.

Apesar dos adsorventes utilizados no processo de separação de n-parafinas serem bastantes conhecidos, como por exemplo, a zeólita $\mathrm{NaCaA}$ (Sun et al., 2008) e a silicalita, nos últimos anos novos materiais e novas rotas de síntese foram descobertos, incluindo ALPO's e SAPO's. Os SAPO's são peneiras moleculares a base de aluminofosfatos apresentando diversas estruturas e composições (Murthy et al., 2001). Os SAPO's, hipoteticamente são produzidos através da substituição de átomos de $\mathrm{Al}$ ou $\mathrm{P}$ pelo $\mathrm{Si}$, numa rede hipotética de aluminofosfatos tridimensionais. Estes materiais foram sintetizados pela Union Carbide em 1984 possuindo características de materiais microporosos com acidez media. Ao mesmo tempo apresentam potencial para serem aplicados no processo de separação de n-parafinas (Wang et al.,
2003) por apresentarem diâmetros seletivos para a separação.

O comportamento estrutural da Zeólita $\mathrm{NaCaA}$ é caracterizado pela presença de microporos sobre a superfície, que se originam no processo de síntese e são extremamente regulares em espaço e tamanho (Auerbach et al., 2003). Desta forma, moléculas podem ser adsorvidas dentro dos seus poros porque seus diâmetros moleculares coincidem com o diâmetro do poro do adsorvente. A Zeólita $\mathrm{NaCaA}$ apresenta diâmetro livre de poro de 4,4 Angstrons, de modo que alcanos lineares cujo diâmetro cinético é de 3,1 Angstrons podem se adsorver dentro da cavidade. A adsorção de hidrocarbonetos leves sobre a Zeólita $\mathrm{NaCaA}$ tem sido estudada em artigos da literatura (Shams, 2007; Sun et al., 2008). Apesar dos adsorventes utilizados no processo de separação de n-parafinas serem bastante conhecidos, nos últimos anos novos materiais e novas rotas de síntese foram descobertos, incluindo ALPO's e SAPOs (Szostak et al., 1998; Martens et al., 1999) Os aluminofosfatos possuem, características de seletividade que possibilitam sua aplicação na separação de n-parafinas, ou seja, diâmetros de poro compatível com as parafinas lineares. Entretanto, dados de adsorção de n-parafinas sobre a superfície desses materiais são escassos na literatura.

Desta forma, o presente trabalho tem como objetivo principal a determinação de isotermas de adsorção do n-undecano e do n-dodecano sobre o SAPO-18, com o intuito de avaliar o potencial desse adsorvente em comparação à zeólita $\mathrm{NaCaA}$.

\section{Materiais e Métodos}

\subsection{Síntese do SAPO-18}

$\begin{array}{crrr}\mathrm{O} & \text { adsorvente } & \text { foi } & \text { sintetizado } \\ \text { utilizando } & \text { como } & & \text { reagentes: }\end{array}$


pseudoboehmita (Sasol, $72 \%$ de pureza); ácido fosfórico (Merck, 85\% de pureza); sílica fumed (Sigma Aldrich); etil-diisopropilamina, direcionador orgânicos (Alfa Aesar) e água destilada. Os reagentes foram adicionados em proporções estequiométricas de modo a se obter uma mistura reacional com a seguinte composição: $1,60 \mathrm{C}_{8} \mathrm{H}_{19} \mathrm{~N}: 0,40 \mathrm{SiO}_{2}: \mathrm{Al}_{2} \mathrm{O}$ $3: 0,9 \mathrm{P}_{2} \mathrm{O}_{5}: 50 \mathrm{H}_{2} \mathrm{O}$ (Chen, 1994).

$\mathrm{O}$ gel de síntese foi mantido sob agitação vigorosa para garantir a homogeneidade e em banho de gelo, a fim de diminuir perdas por evaporação do etildiisopropilamina provocada pelo ao aumento de temperatura, já que o processo é exotérmico. Em seguida a mistura reacional foi submetida a um pré-aquecimento em autoclave a $195^{\circ} \mathrm{C}$, durante 8 dias. Ao fim do tempo de cristalização a amostra obtida foi lavada por filtração a vácuo, até $\mathrm{pH}$ neutro da água de lavagem. $\mathrm{O}$ filtrado foi condicionado em estufa pré-aquecida a $55^{\circ} \mathrm{C}$ por mais 16 horas.

A calcinação do SAPO-18 foi realizada em uma mufla pré-aquecida a $500{ }^{\circ} \mathrm{C}$ durante 20 horas (Silva, 1999). A taxa de aquecimento utilizada foi de 5 ${ }^{\circ} \mathrm{C} \min ^{-1}$ sob o fluxo de $\mathrm{N}_{2}$ de $60 \mathrm{~mL}$ $\min ^{-1}$, sendo em seguida substituído por ar sintético, mantendo-se a mesma vazão e temperatura.

\subsection{Síntese da Zeólita $\mathrm{NaCaA}$}

A forma sódica da zeólita A foi sintetizada pelo método hidrotérmico utilizando os seguintes reagentes: Aluminato de Sódio (54\% de $\mathrm{Al}_{2} \mathrm{O}_{3}$; $41 \% \mathrm{Na}_{2} \mathrm{O} ; 5 \% \mathrm{H}_{2} \mathrm{O}$ ); Sílica Aerosil; Hidróxido de Sódio $(57,5 \%)$ e água destilada. A relação molar utilizada para o cálculo das quantidades dos reagentes utilizados na síntese da zeólita $\mathrm{NaCaA}$ foi de: $\mathrm{SiO}_{2} / \mathrm{Al}_{2} \mathrm{O}_{3}=2 ; \mathrm{Na}_{2} \mathrm{O} / \mathrm{SiO}_{2}=$ 2,25; $\mathrm{H}_{2} \mathrm{O} / \mathrm{Na}_{2} \mathrm{O}=30$ (Shams, 2007). O Hidróxido de Sódio foi dissolvido em água destilada e, em seguida, o
Aluminato de Sódio e a Sílica Aerosil foram adicionados lentamente, mantendo-se agitação constante e temperatura entre 70 e $80^{\circ} \mathrm{C}$, durante três horas. A agitação e o aquecimento foram interrompidos após a formação do gel de síntese e o mesmo permaneceu em repouso durante 72 horas para o processo de nucleação. Para o processo de cristalização, o gel de síntese foi submetido a um novo procedimento de agitação a temperatura de $90^{\circ} \mathrm{C}$ por mais 3,5 horas. $\mathrm{O}$ cristal da Zeólita NaA, precipitado no fundo do recipiente, foi separado através de filtração, submetido a lavagens sucessivas até o filtrado atingir $\mathrm{pH}$ menor que 11 e seco em estufa a $150^{\circ} \mathrm{C}$, durante $12 \mathrm{~h}$. $\mathrm{O}$ material obtido foi submetido à troca iônica com uma solução de cloreto de cálcio $1 \mathrm{M}$, por um período de $24 \mathrm{~h}$ a temperatura ambiente. Mais adiante, a solução de cloreto de cálcio foi substituída por uma mais nova e mantida a $70{ }^{\circ} \mathrm{C}$ durante mais $4 \mathrm{~h}$. A Zeólita $\mathrm{NaCaA}$ foi separada através de filtração a vácuo (Shams e Mirmohammadi, 2007).

\subsection{Caracterização}

Os adsorventes foram caracterizados através de Difração de Raios-X (DRX), Fluorescência de Raios-X (FRX), Análise Termogravimétrica (TG) e Adsorção de $\mathrm{N}_{2}$ a $-196{ }^{\circ} \mathrm{C}$. As análises de DRX foram realizadas em um difratômetro de raios X XRD - 6000 SHIMADZU utilizando um tubo de cobre (radiação $\mathrm{K} \alpha$ ) com um ângulo de varredura entre 5 e $50^{\circ}$ com uma velocidade de 2 graus por min. As medidas de adsorção de $\mathrm{N}_{2}$ a $-196{ }^{\circ} \mathrm{C}$ foram realizadas no medidor de área superficial Micromeritics, modelo ASAP-2020. A massa de adsorvente utilizada na análise foi de 0,2 g aproximadamente. Para varredura completa dos elementos dos adsorventes foi realizada uma análise de 
Fluorescência de Raios-X (FRX) no equipamento Shimadzu modelo XRF1800. A técnica de FRX foi empregada utilizando pastilhas prensadas de ácido bórico com o material a ser analisado. $\mathrm{O}$ estudo de decomposição térmica foi realizado através da análise termogravimétrica (TGA) em um equipamento SHIMADZU DTG-60H Simultaneous DTA-TG. Para realização desse ensaio, estabeleceu-se uma taxa de aquecimento de $5^{\circ} \mathrm{C} / \mathrm{min}$ até a temperatura máxima $1000{ }^{\circ} \mathrm{C}$.

\subsection{Dados de Equilíbrio}

Para a determinação dos dados de equilíbrio os seguintes reagentes foram utilizados: n-undecano $99 \%$ de pureza Merck; n-dodecano 99\% de pureza Merck e cicloexano 99\% de pureza Merck. Os experimentos foram realizados utilizando amostras de SAPO-18 e zeólita NaCaA. Na etapa de pré-tratamento as amostras foram condicionadas em estufa durante 4 horas, a $200{ }^{\circ} \mathrm{C}$, para eliminar qualquer umidade que pudesse interferir nos resultados. Amostras de adsorvente $(0,05 \mathrm{~g})$ foram colocadas em vials selados com septo para evitar perdas por evaporação, contendo em torno de $1 \mathrm{~g}$ de solução (n-parafina e cicloexano). As soluções iniciais foram preparadas com concentrações diferentes de n-parafina (C11 e C12) em cicloexano variando de 2 a $35 \%$. Os recipientes permaneceram em repouso durante 24 horas em banho termostatizado a temperatura controlada. O cicloexano foi escolhido como adsortivo para representar a fase da mistura que não tem afinidade pela superfície dos adsorventes, já que o diâmetro cinético da molécula é maior que o diâmetro do poro dos dois adsorventes. Após o equilíbrio ser atingido, alíquotas da solução em equilíbrio com o adsorvente, contendo $0,5 \mu \mathrm{L}$ foram coletadas e injetadas em um cromatógrafo a gás HP 5890 series
II equipado com detector FID e coluna capilar do tipo OV1. As análises foram realizadas em triplicata com erro de no máximo $5 \%$. A quantidade de nparafina adsorvida nos adsorventes foi calculada pelo balanço de massa através da equação 1 (Chempath et al., 2004).

$$
q=\frac{m_{\text {solução } *\left(C_{0}-C_{f}\right)}}{m_{\text {Adsorvente }}}
$$

Em que:

$C_{0}$ é a concentração inicial de parafina/cicloexano; $C_{f}$ é a concentração final de parafina/cicloexano após o equilíbrio ter sido alcançado; $m_{\text {solução }}$ é a massa total de parafina/cicloexano; $m_{\text {Adsorvente }}$ é a massa de adsorvente utilizada para obtenção dos dados de equilíbrio.

O modelo de Langmuir (Equação 2) foi utilizado para ajustar os dados de equilíbrio de adsorção das parafinas sobre os adsorventes (Ruthven, 1984).

$$
q=\frac{q_{m} * K * C}{(1+K * C)}
$$

Em que:

$q$ é a quantidade adsorvida (g de parafina/g de adsorvente); $q_{m}$ é a capacidade máxima de adsorção (g de parafina/g de adsorvente); $K$ é a constante de equilíbrio e $C$ é a concentração de equilíbrio.

\section{Resultados e Discussão}

\subsection{Caracterização}

A Figura 1 apresenta 0 difratograma de difração de Raios-X da zeólita $\mathrm{NaA}$. 


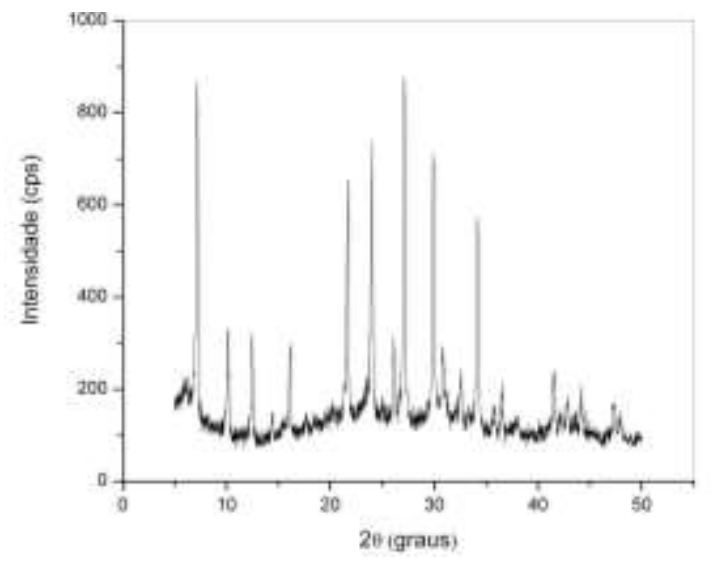

Figura 1. Difração de Raios- $X$ da zeólita NaA.

Os picos apresentados na Figura 1 são característicos da estrutura da zeólita $\mathrm{NaA}$, conforme apresentado na literatura (Sun et al., 2008; Reed e Breck, 1956; Gramlich e Meier, 1971). Após a troca iônica, análises de fluorescência de Raios-X foram realizadas para avaliar a troca iônica de parte dos íons sódio por íons cálcio. A composição da zeólita $\mathrm{NaCaA}$ é mostrada na Tabela 1.

A Figura 2 apresenta o resultado da análise de Difração de Raios-X para o SAPO-18. A amostra obtida pelo método hidrotérmico apresentou-se homogênea sem a presença de fases secundárias e os picos de difração estão em concordância com o apresentado na literatura (Muñoz et al., 2012).

Tabela 1. Fluorescência de Raios- $X$ da zeólitaNaCaA.

\begin{tabular}{c|c}
\hline Componente & $\begin{array}{c}\text { Percentual em peso } \\
(\%)\end{array}$ \\
\hline $\mathrm{SiO}_{2}$ & 46,95 \\
$\mathrm{Al}_{2} \mathrm{O}_{3}$ & 38,49 \\
$\mathrm{CaO}$ & 11,7 \\
$\mathrm{Na}_{2} \mathrm{O}$ & 2,17 \\
$\mathrm{TiO}_{2}$ & 0,39 \\
$\mathrm{Fe}_{2} \mathrm{O}_{3}$ & 0,19 \\
$\mathrm{~K}_{2} \mathrm{O}$ & 0,09 \\
\hline
\end{tabular}

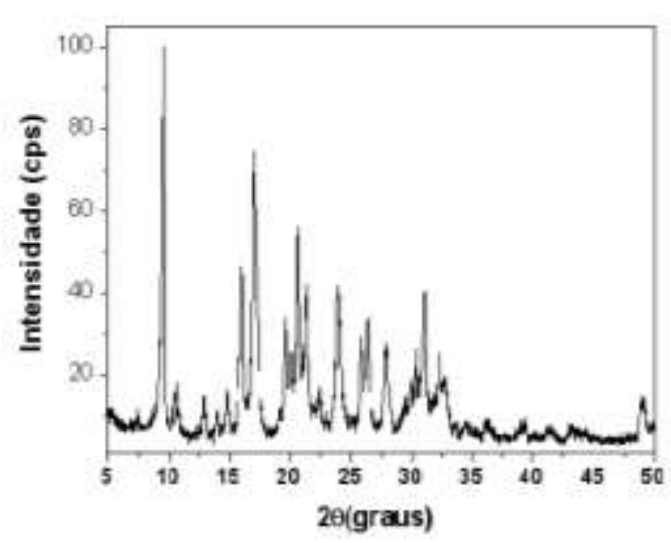

Figura 2. Difratograma de Raios-X do SAPO-18.

A Figura 3 representa a adsorção de nitrogênio sobre o SAPO-18.

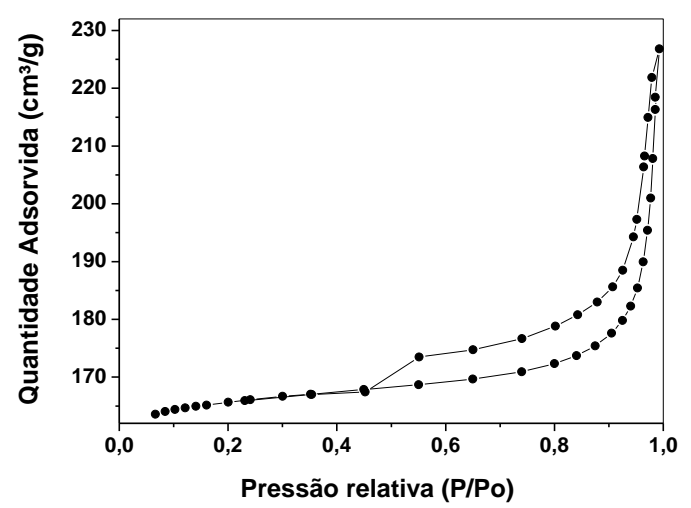

Figura 3. Adsorção de nitrogênio sobre o SAPO-18.

$\mathrm{O}$ formato da isoterma de adsorção do nitrogênio sobre a superfície do SAPO-18 corresponde a um comportamento típico de materiais microporosos (Gregg e Sing, 1982). A região da isoterma de adsorção da Figura 3, compreendida entre os valores de $\mathrm{P} / \mathrm{P}_{0}$ de 0 e 0,2 (baixas pressões), é caracterizada pela adsorção em monocamada. Já o comportamento a médias pressões é característico da adsorção em multicamadas, como é mostrado na região da isoterma com baixa inclinação, no intervalo de $\mathrm{P} / \mathrm{P}_{0}$ de 0,4 a 0,8 . Na região de altas pressões relativas não existe qualquer adsorção limitante, o que é uma evidência de que o adsorvente não possui estrutura mesoporosa bem definida. A presença 
da histerese está associada ao processo de condensação capilar nos mesoporos.

Por se tratar de um material microporoso, os dados da isoterma de $\mathrm{N}_{2}$ a $-196{ }^{\circ} \mathrm{C}$ sobre o SAPO-18 foram ajustados pelo método t-plot, conforme mostrado na Figura 4.

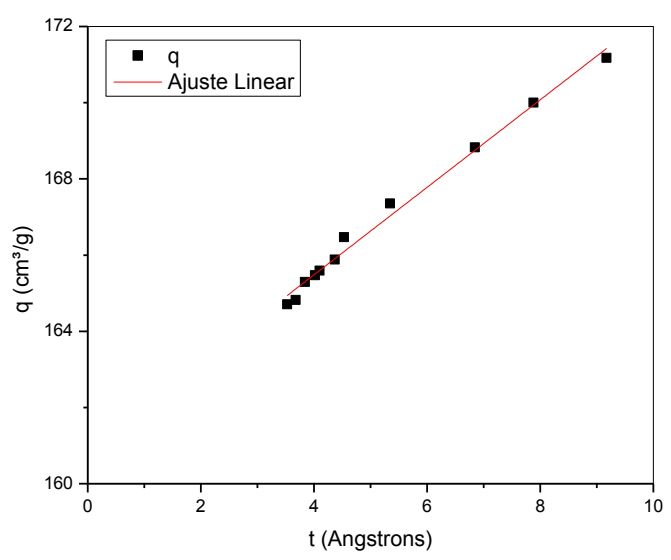

Figura 4. t-plot da isoterma de $\mathrm{N}_{2}$ a $196{ }^{\circ} \mathrm{C}$ sobre o SAPO- 18 .

Os parâmetros obtidos a partir do ajuste do método t-plot (Lippens e de Boer, 1965) estão mostrados na Tabela 2.

Tabela 2. Parâmetros do modelo t-plot.

\begin{tabular}{c|c}
\hline Coeficiente de & 0,9894 \\
correlação $\left(\mathrm{R}^{2}\right)$ & \\
Inclinação & 1,1485 \\
Coeficiente linear & 160,89 \\
Área superficial $\left(\mathrm{m}^{2} / \mathrm{g}\right)$ & 17,77 \\
Volume de micro poro & 0,249 \\
$\left(\mathrm{~cm}^{3} / \mathrm{g}\right)$ & \\
\hline
\end{tabular}

Para o cálculo da espessura $t$ foi utilizada a Equação 3 (Harkins e Jura, 1944).

$$
t=\left[\frac{13,99}{0,034-\log \left(\frac{P}{P_{0}}\right)}\right]^{0,5}
$$

A partir da análise termogravimétrica do SAPO-18, apresentada na Figura 5, foi possível determinar que a $400^{\circ} \mathrm{C}$ ocorre uma perda de massa relativa de 13,68\% referente ao "template"; o direcionador orgânico. A análise termogravimétrica também garantiu a utilização do SAPO18 como adsorvente no processo de separação de n-parafinas sem danos na sua estrutura cristalina, com uma temperatura de ativação de $350{ }^{\circ} \mathrm{C}$.

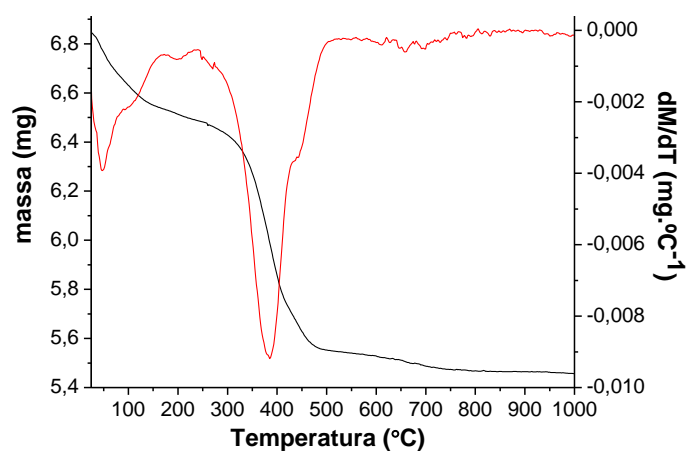

Figura 5. Análise termogravimétrica derivativa do SAPO-18.

\subsection{Isotermas de Adsorção}

As Figuras 6 e 7 apresentam a isoterma de adsorção do n-undecano em cicloexano sobre a zeólita $\mathrm{NaCaA}$ e o SAPO-18, respectivamente, ambas obtidas em fase líquida a temperatura de $25^{\circ} \mathrm{C}$.

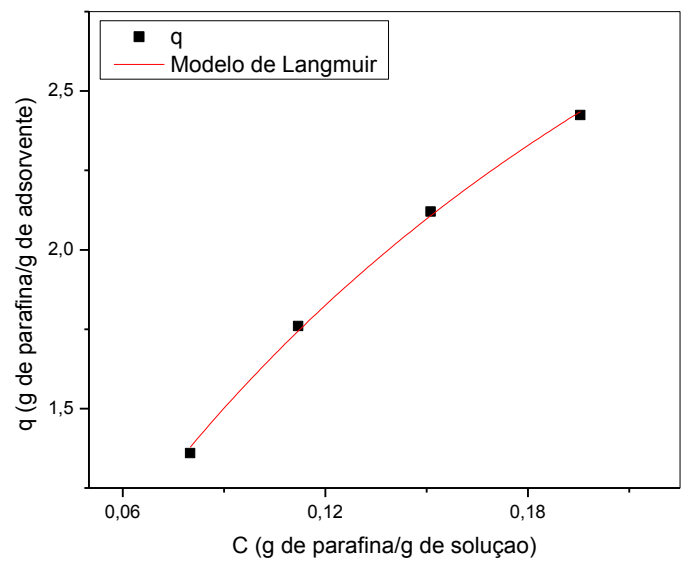

Figura 6. Isoterma de adsorção do nundecano sobre a zeólita $\mathrm{NaCaA}$ ajustada pelo modelo de Langmuir. 


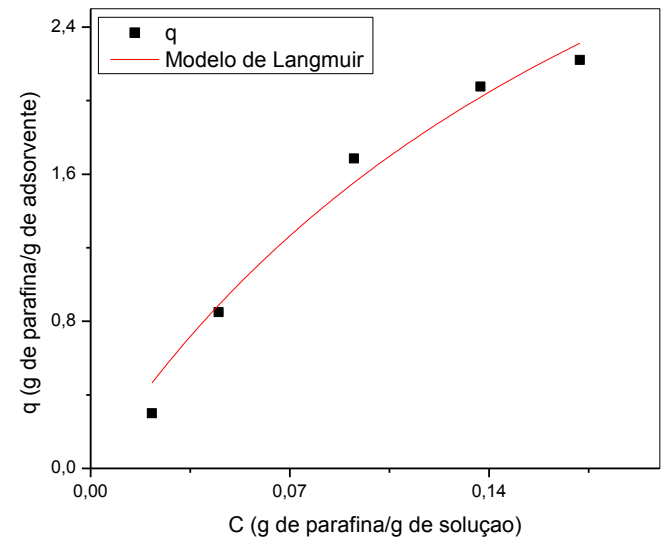

Figura 7. Isoterma de adsorção do nundecano sobre o SAPO-18 ajustada pelo modelo de Langmuir.

As isotermas apresentadas foram determinadas em temperatura ambiente para a zeólita $\mathrm{NaCaA}$ e para o SAPO18. É possível observar que a forma das isotermas de adsorção das parafinas sobre o SAPO-18 apresenta comportamento semelhante ao da zeólita $\mathrm{NaCaA}$. Essa semelhança de comportamento se deve, em parte, às características da estrutura dos materiais. Como o processo de adsorção nesse caso é de origem física regido pelo mecanismo de peneiramento molecular e os dois adsorventes, SAPO18 e zeólita $\mathrm{NaCaA}$, apresentam aproximadamente o mesmo diâmetro de poro, tal comportamento é esperado. $\mathrm{O}$ mesmo pode ser dito para as isotermas do n-dodecano sobre a zeólita $\mathrm{NaCaA}$ e o SAPO-18 apresentadas nas Figuras 8 e 9 , respectivamente.

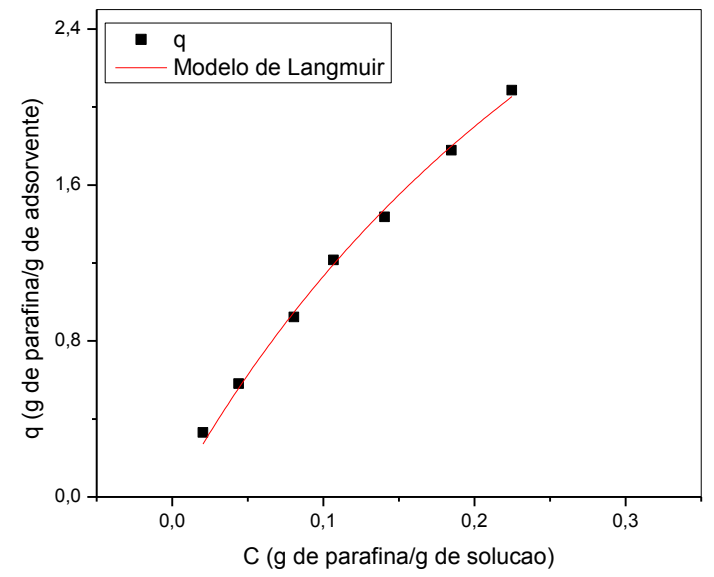

Figura 8. Isoterma de adsorção do ndodecano sobre a zeólita $\mathrm{NaCaA}$ ajustada pelo modelo de Langmuir.

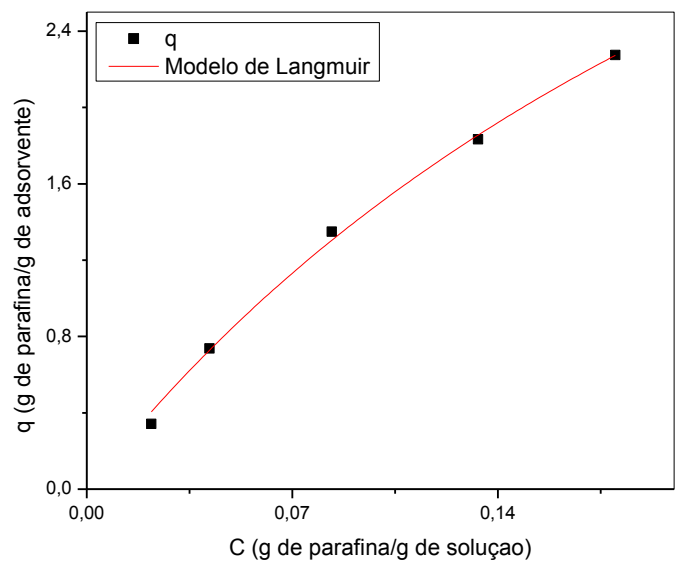

Figura 9. Isoterma de adsorção do ndodecano sobre o SAPO-18 ajustada pelo modelo de Langmuir.

As Tabelas 3 e 4 apresentam os dados das isotermas do n-undecano e ndodecano sobre os adsorventes.

Tabela 3. Dados das isotermas do nundecano sobre os adsorventes estudados.

\begin{tabular}{cccc}
\hline \multicolumn{2}{c}{$\mathrm{NaCaA}$} & \multicolumn{2}{c}{$\mathrm{SAPO}-18$} \\
\hline $\mathrm{C}(\%)$ & $\mathrm{q}(\mathrm{g} / \mathrm{g})$ & $\mathrm{C}(\%)$ & $\mathrm{q}(\mathrm{g} / \mathrm{g})$ \\
0,080 & 1,360 & 0,021 & 0,300 \\
0,112 & 1,760 & 0,045 & 0,850 \\
0,151 & 2,120 & 0,092 & 1,686 \\
0,195 & 2,424 & 0,137 & 2,076 \\
& & 0,172 & 2,222 \\
\hline
\end{tabular}


O código utilizado na regressão não linear dos dados das isotermas de adsorção foi baseado no método GaussNewton. As isotermas foram ajustadas pelo modelo clássico de Langmuir, conforme a Equação 2. O coeficiente de correlação $\left(\mathrm{R}^{2}\right)$ encontrado para a zeólita $\mathrm{NaCaA}$ foi de 0,9979 e a capacidade máxima de adsorção, $\mathrm{q}_{\mathrm{m}}$ igual a $5,19 \mathrm{~g} / \mathrm{g}$. Já para o SAPO-18 o $\mathrm{R}^{2}$ foi de 0,9719 e o $\mathrm{q}_{\mathrm{m}}, 5,36 \mathrm{~g} / \mathrm{g}$.

Tabela 4. Dados das isotermas do ndodecano sobre os adsorventes estudados.

\begin{tabular}{cccc}
\hline \multicolumn{2}{c}{$\mathrm{NaCaA}$} & \multicolumn{2}{c}{ SAPO-18 } \\
\hline $\mathrm{C}(\%)$ & $\mathrm{q}(\mathrm{g} / \mathrm{g})$ & $\mathrm{C}(\%)$ & $\mathrm{q}(\mathrm{g} / \mathrm{g})$ \\
0,020 & 0,331 & 0,022 & 0,342 \\
0,044 & 0,581 & 0,042 & 0,737 \\
0,081 & 0,923 & 0,083 & 1,349 \\
0,107 & 1,215 & 0,133 & 1,833 \\
0,140 & 1,436 & 0,180 & 2,275 \\
0,184 & 1,778 & & \\
0,225 & 2,086 & & \\
\hline
\end{tabular}

O mesmo procedimento descrito para as isotermas do n-undecano foi realizado para o n-dodecano. $\mathrm{O}$ coeficiente de correlação $\left(\mathrm{R}^{2}\right)$ encontrado para a zeólita $\mathrm{NaCaA}$ foi de 0,9958 e a capacidade máxima de adsorção, $\mathrm{q}_{\mathrm{m}}$ igual a 5,9 g/g. Já para o SAPO-18 o $\mathrm{R}^{2}$ foi de 0,9964 e o $\mathrm{q}_{\mathrm{m}}$, $6,35 \mathrm{~g} / \mathrm{g}$. A Tabela 5 apresenta o comparativo dos valores de $\mathrm{q}_{\mathrm{m}}$ para as duas parafinas e os dois adsorventes.

Tabela 5. Valores de $\mathrm{q}_{\mathrm{m}}(\mathrm{g} / \mathrm{g})$ para o $\mathrm{n}$ undecano e n-dodecano sobre a zeólita $\mathrm{NaCaA}$ e o SAPO-18.

\begin{tabular}{lcc}
\hline & NaCaA & SAPO-18 \\
\hline n-undecano & 5,19 & 5,36 \\
n-dodecano & 5,90 & 6,35 \\
\hline
\end{tabular}

É possível observar a partir da Tabela 5 que a capacidade máxima de adsorção das parafinas foi maior para o SAPO-18, comprovando assim o potencial desse adsorvente para ser aplicado no processo de separação de nparafinas de correntes de refino.

\section{Conclusão}

Foi apresentada uma rota de síntese para o SAPO-18 e a análise de Difração de Raios-X comprovou a estrutura. A zeólita $\mathrm{NaCaA}$ foi sintetizada para ser comparada ao cristal do SAPO-18. O formato da isoterma de nitrogênio a $-196^{\circ} \mathrm{C}$ encontrado para o SAPO-18 é característico de um material microporoso. A TG garantiu que o SAPO-18 será estável termicamente quando submetido a 400 ${ }^{\circ} \mathrm{C}$ no processo de separação de nparafinas. O formato das isotermas é característico de adsorção sobre materiais microporosos e trabalhos anteriores confirmaram que a isoterma segue o padrão de Langmuir, conforme apresentado. O SAPO-18 apresentou maior capacidade de adsorção para as duas parafinas estudadas.

\section{Referências}

AUERBACH, S. M., CARRADO, K. A., DUTTA, P. K. Handbook of Zeolite Science and Technology. Marcel Dekker, Inc. 2003

CHEMPATH, S.; DENAYER, J. F. M.; DE MEYER, K. M. A.; BARON, G. V.; SNURR, R. Q. Adsorption of LiquidPhase Alkane Mixtures in Silicalite: Simulations and Experiment. Langmuir 2004, 20, 150-156.

CHEN, J. SAPO-18 catalysts and their Bronsted acid sites. The Royal Institution of Great Britain. London, 1994.

D.M. RUTHVEN, Principles of Adsorption and Adsorption Processes, John Wiley, New York, 1984. 
GRAMLICH, V. AND MEIER, W.M. The crystal structure of hydrated $\mathrm{NaA}$ : A detailed refinement of a pseudosymmetric zeolite structure. Z. Kristallogr., 133, 134-149 (1971).

GREGG S. J.,SING K. S. W. Adsorption, Surface Area and Porosity. $2^{\text {a }}$ Ed. London: Academic Press. 1982.

HARKINS, W. D.; JURA, G; J. Am. Chem. Soc. 1944, 66, 1366.

J. A. MARTENS, P. A. JACOBS, in: J. Weitkamp, L. Puppe (Eds.), Catalysis and Zeolites, Springer Verlag, Berlin, Heidelberg, New York, 1999, p. 53.

TEJERO, J. L. B., ALMEIDA, J. L. G., SERRANO, I. L. Process to obtain a highly soluble linear alkylbenzenesulfonate.US 8158819 B2. 2012.

WANG, L., GUO, C., YAN, S., HUANG, X, Li, Q. High-silica SAPO-5 with preferred orientation: synthesis, characterization and catalytic applications. Microporous and Mesoporous Materials 64 (2003) 63-68.

LIRA, A; TAILLEUR, R. G. Dehydrogenation of C12-C14 paraffins on $\mathrm{PtCu} /$ meso-structured $\mathrm{Al}_{2} \mathrm{O}_{3}$ catalyst for $\mathrm{LAB}$ production: Process simulation. Fuel 97 (2012) 49-60.

LIPPENS B.C., AND DE BOER J.H., 1965, J. Catal. 4, 319.

MURTHY, K.V.V.S.B.S.R. ; KULKARNI, S.J.; MASTHAN, S.K. Sorption properties of modified silicoaluminophosphate SAPO-5 and
SAPO-11 molecular sieves. Microporous and Mesoporous Materials 43 (2001) 201-209.

MUÑOZ, T. A., ÁlVAREZ, C. M., ENRIQUE SASTRE, E. Synthesis of sapo-34 and sapo-18 with enhanced properties for the methanol transformation to olefins. Av. cien. ing.: 3(2), 47-56 (Abril/Junio, 2012).

REED, T.B. AND BRECK, D.W.CRYSTALLINE ZEOLITES. II. Crystal structures of synthetic zeolite, type A. J. Am. Chem. Soc., 78, 59725977 (1956).

R. SZOSTAK, in: H.G. Karge, J. Weitkamp (Eds.), MolecularSieves Science and Technology, Vol. 1, Springer Verlag,Berlin, Heidelberg, New York, 1998, p. 157.

SHAMS, K.; MIRMOHAMMADI, S.J. Preparation of 5A zeolite monolith granular extrudates using kaolin: Investigation of the effect of binder on sieving/adsorption properties using a mixture of linear and branched paraffin hydrocarbons. Microporous and Mesoporous Materials 106 (2007) 268277.

SILVA, A.O.S. Síntese, caracterização e propriedades catalíticas de aluminofosfatos contendo nióbio. Dissertação (Mestrado) - UFRN, Natal, 1999.

SUN, H.; BENXIAN, S.; JICHANG, L. N-Paraffins adsorption with 5A zeolites: The effect of binder on adsorption equilibria. Separation and Purification Technology 64 (2008) 135-13. 